\title{
Cardiac Resynchronization Therapy using Left-Bundle-Branch Area and Left Ventricular Pacing
}

Xiang-Fei Feng ${ }^{1}$, Ling-Chao Yang ${ }^{1}$, Rui Zhang ${ }^{2}$, Yi Yu², Bo Liu ${ }^{1}$, Ya-Qin Han², Qiu-Fen $\mathrm{Lu}^{1}$, and Yi-Gang $\mathrm{Li}^{1}$

${ }^{1}$ Xinhua Hospital, Shanghai Jiao Tong University School of Medicine, China.

${ }^{2}$ Xinhua Hospital Affiliated to Shanghai Jiaotong University School of Medicine

December 2, 2020

\begin{abstract}
Introduction: Cardiac resynchronization therapy via biventricular pacing is an established therapy for patients with heart failure. However, high nonresponder rates and inability to predict response remains a challenge. Recently left bundle branch area pacing (LBBAP) has been shown to be feasible and may also improve clinical outcomes. In this article we describe sequential LBBAP followed by left ventricular (LV) pacing (LOT-CRT) and assess the feasibility of LOT-CRT. Methods: The RV implantation site was positioned and the LBBAP lead was implanted using our methods. The QRS duration (QRSd) at baseline, during LBBAP, biventricular pacing, and LOT-CRT was measured. Results: LOT-CRT was successful in 5 patients (age 71.8 \pm 5.1 years, men 3, ischemic 3). The QRSd at baseline was $158.0 \pm 13.0 \mathrm{~ms}$ and significantly narrowed to $117.0 \pm 6.7$ ms during LOT-CRT $(\mathrm{P}<0.01)$. During 3-month follow-up, LV ejection fraction improved from $32.8 \pm 5.2 \%$ to $45.0 \pm 5.1 \%$ $(\mathrm{P}<0.01)$, and New York Heart Association functional class changed from $3.25 \pm 0.5$ to $2.5 \pm 0.6(\mathrm{P}<0.05)$. A decrease in left ventricular end-diastolic dimension was observed, with widening from $(68.2 \pm 12.3) \mathrm{mm}$ at baseline to $(62.2 \pm 11.3) \mathrm{mm}$ at pacing $(\mathrm{P}<0.05)$. The length of operation time was $(152.0 \pm 31.1)$ min. Conclusions: The study demonstrates that LOT-CRT is clinically feasible in patients with systolic HF and LBBB. LOT-CRT was associated with significant narrowing of QRSd and improvement in LV function, especially in patients with ischemic cardiomyopathy.
\end{abstract}

\section{Cardiac Resynchronization Therapy using Left-Bundle-Branch Area and Left Ventricular Pa- cing}

Xiang-Fei Feng, MD\#, Ling-Chao Yang, PhD\#, Rui Zhang, MA, Yi Yu, PhD, Bo Liu, PhD, Ya-Qin Han, BA, Qiu-Fen Lu, MA, Yi-Gang Li, MD

Department of Cardiology, Xinhua Hospital, School of Medicine, Shanghai Jiao Tong University, Shanghai 200092, China.

\#Xiang-Fei Feng and Ling-Chao Yang contributed equally to this study as first authors.

Address for correspondence:

Xiang-Fei Feng and Yi-Gang Li are joint corresponding authors.

Yi-Gang Li, MD

Department of Cardiology, Xinhua Hospital, School of Medicine, Shanghai Jiao Tong University, 1665\#, KongJiang Road, Shanghai 200092, China

Tel: (001) 86-21-55964561; Fax: (001) 86-21-25077275

E-mail: drliyigang@yeah.net; fengxiangfei@xinhuamed.com.cn 
Running title: LOT-CRT

Total word count: 3478

The total numbers of tables and figures: (tables: 3, figures: 2)

Disclosures: None.

\section{INTRODUCTION}

Cardiac resynchronization therapy (CRT) via biventricular pacing (BVP) is known to improve clinical outcomes, and decreases all-cause mortality, particularly in patients with left bundle branch block (LBBB), and reduced left ventricular function ${ }^{1,2}$. Permanent His bundle pacing (HBP) has been shown to overcome $\mathrm{LBBB}$, and is being considered as a viable alternative to BVP in patients requiring $\mathrm{CRT}^{3}$. However, HBP may be associated with high pacing thresholds to capture the distal His bundle and/or correct $\mathrm{LBBB}^{4}$.

Recently several groups have shown the feasibility of left bundle branch area pacing (LBBAP) as an alternative choice to HBP in patients with LBBB by pacing the LBB region beyond the block site with a stable threshold and narrow QRS duration (QRSd) $)^{5-7}$. However, it is unknown whether the clinical efficacy of LBBAP with an appropriate AV delay would be the same as or better than LV epicardial pacing or cardiac resynchronization therapy.

We hypothesized that electrical resynchronization measured by narrowing of the QRS complex can be accomplished more effectively by LBBAP followed by sequential LV pacing (LBB-Optimized LV pacing, LOTCRT). The aim of our study is to assess the feasibility and efficacy of LOT-CRT to improve electrical resynchronization in patients qualifying for CRT and evaluate clinical and echocardiographic response rates.

\section{METHODS}

This study was approved by Ethics Committee of Xinhua Hospital Affiliated to Shanghai Jiao tong University School of Medicine (approval number: XHEC-D-2020-148) and performed in accordance with the Declaration of Helsinki.

\subsection{Patient's selection}

Patients with chronic LBBB by Strauss criteria ${ }^{8}$, optimal medical therapy-refractory New York Heart Association (NYHA) class III to IV heart failure(HF) symptoms and a baseline left ventricular ejection fraction (LVEF, calculated by Simpson method) [?] 35\% were enrolled in Shanghai Xinhua Hospital from April 1, 2019 to June 1, 2020.

Patients were excluded if they had a history of previous valve intervention, end-stage renal disease, previous heart transplantation, left ventricular assist device, metastatic cancer, moderate or severe valve disease, life-expectancy less than 1 year.

All patients submitted written informed consent and demonstrated an understanding of LOT-CRT as a nonstandard approach to achieve physiologic pacing, and their data were analyzed prospectively.

\subsection{Procedural Details}

The RV defibrillator lead was first implanted in the RV to provide backup ventricular pacing should the patient develop transient complete atrioventricular block during the LBBAP lead placement. Subsequently, the LV lead was implanted in the standard fashion targeting sites with maximal LV delay. Then, LBBAP was performed using the Select Secure pacing lead. The fluoroscopy duration for the entire procedure, LBBAP lead implant and LV lead implant were separately recorded.

\subsection{LBBAP lead implantation technique}

As previously described ${ }^{9-12}$, a Select Site C315 His sheath and a Select Secure 3830 pacing lead (Medtronic Inc, Minneapolis, MN, USA) were advanced to the implantation site. Right ventricular septal location for LBBAP is identified using the anatomical location and pacing localization in nine-grid system ${ }^{13}$. Once 
this site is identified, the pacing lead is advanced deep into the septum while monitoring unipolar pacing impedance, electrogram characteristics and paced QRS morphology.

Additionally, the lead orientation can be displayed in various projections. During the initial LBBAP lead fixation, if the lead torques back, it will mean that the lead and sheath are not oriented orthogonal to the RV septum. Generally, the sheath and the lead are oriented such that the lead is pointing towards 12-1 O'clock direction right anterior oblique $30^{\circ}$ view and 2-3 O'clock direction in left anterior oblique $30^{\circ}$ view ${ }^{14}$.

In addition to fluoroscopic views, significant rise in unipolar pacing impedance above 900 Ohms would suggest that the lead is directing to an oblique direction and need re-oriented. If several attempts were made to achieve LBBAP, prior sites were tagged in the mapping system or fluoroscopic view to prevent re-engaging in the same site. Once the lead position was finalized, 1-2 $\mathrm{ml}$ of contrast was injected through the delivery sheath to visualize the septal wall and the approximate depth of the lead under left anterior oblique fluoroscopic view.

\subsection{Device Connection and Programming}

In patients with normal sinus rhythm undergoing CRT-defibrillators, the LBBAP lead was connected to the pace-sense portion of RV port and a bipolar LV lead in the LV port. The pace-sense portion of the spliced ICD lead (DF-1) was capped. In patients with normal sinus rhythm undergoing CRT-pacemakers (P), the LBBAP lead was connected to the RV port and the LV lead to the LV port.

\subsection{Implant Measurements}

The pacing output required to maximally narrow the QRS (BBB correction threshold) and LBB capture threshold (without BBB correction) was assessed and recorded at a pulse width of $1.0 \mathrm{~ms}$. The QRSd at baseline, during LBBAP, BVP (via RV defibrillator lead, when available) and LBB-Optimized LV pacing were measured on the EP recording system at $100 \mathrm{~mm} / \mathrm{s}$. The interval from the onset of QRS to the maximal deflection of LV electrogram (LVAT) during native LBBB pacing and during LBBAP was documented.

\subsection{Clinical follow-up}

Patients were seen for routine clinical follow-up at standard time periods (every 3 months). Functional status was assessed by NYHA classification. Device thresholds were checked and adjusted as needed to maximize battery longevity. The pacing threshold, impedance and $\mathrm{R}$ wave amplitude were measured. According to previous literature ${ }^{15}$, the high pacing threshold was defined as pacing threshold over $2.5 \mathrm{~V} / 0.4 \mathrm{~ms}$, increased threshold over $1.0 \mathrm{~V}$ compared with the baseline after the procedure and at follow-up. Echocardiographic indices, including LVEF, LV end-diastolic dimensions, pulmonary artery systolic pressure were recorded pre-implant and at follow-up.

\subsection{Statistical analysis}

Continuous variables were given as mean $\pm \mathrm{SD}$ or median. Paired comparisons were made using a Student $\mathrm{t}$ test if the data were normally distributed, and with the Wilcoxon signed-rank test for nonparametric data. Paired categorical data (NYHA functional class) were compared using the Wilcoxon test. P [?] 0.05 was considered significant.

\section{RESULTS}

Seven out of the 12 screened patients were excluded from the study according to the exclusion criteria. Consequently, 5 patients were registered into the LOT-CRT cohort.

\subsection{Baseline characteristics}

Among 5 patients, three patients $(60 \%)$ were male. All patients had cardiomyopathy (2 with nonischemic and 3 with ischemic), and 2 patients had paroxysmal atrial fibrillation. Hypertension was present in all the patients. The mean age was $71.8+-5.1$ years, and the baseline characteristics of patients are provided in 
Table 1. The baseline LVEF and the baseline QRSd with LBBB were $32.8+-5.2 \%$ and $158.0+-13.0 \mathrm{~ms}$ respectively (Figure 1A).

All patients had at least $1 \mathrm{HF}$ hospitalization 3 months before LBBAP implantation. ENTRESTO (sacubitril / valsartan), $\beta$-blockers, and loop diuretics were prescribed in all patients.

\subsection{Procedural Outcomes}

LOT-CRT was successfully achieved in all 5 patients. The acute success rate was $100 \%$. The operation duration was $152.0 \pm 31.1 \mathrm{~min}$. The time of X-ray fluoroscopy was $26.2 \pm 5.9 \mathrm{~min}$. Biventricular ICD (CRTD) was implanted in 4 patients (Figure 2A, 2B) and CRT-pacemakers in the remaining 1 patient (Table 1).

Both LBBAP and LV capture thresholds remained stable during follow-up $(1.3 \pm 0.6 \mathrm{~V}$ at $0.4 \mathrm{~ms}$ vs $1.6 \pm$ $0.7 \mathrm{~V}$ at $0.4 \mathrm{~ms}$ ). Bipolar LBBAP resulted in partial, but significant narrowing of QRSd (BBB correction) in 5 patients (Figure 1B).

\subsection{ECG characteristics and pacing parameters}

Individual electrocardiographic responses to RV, LV, and LBBAP at the time of implantation are shown in Table 2. Among 5 patients, the baseline QRSd was $158.0 \pm 13.0 \mathrm{~ms}$. After unipolar LBBAP, 5 patients demonstrated an RBBB pattern with a paced QRSd of $123.0 \pm 5.7 \mathrm{~ms}(\mathrm{P}=0.001$ vs. baseline). LBB potential could be recorded in 3 patients from the LBB lead $(60 \%)$. The LVAT for all LBBAP patients was $72.5 \pm 9.4 \mathrm{~ms}$, and the $\mathrm{R}$ wave amplitude, pacing impedance, and unipolar pacing capture threshold were $9.9 \pm 7.2 \mathrm{~V}, 678 \pm 102$, and $0.84+-0.17 \mathrm{~V} / 0.4 \mathrm{~ms}$ respectively after implantation.

BVP resulted in significant narrowing of QRSd from $158.0+-13.0 \mathrm{~ms}$ at baseline to $132.0+-4.5 \mathrm{~ms}$ $(\mathrm{P}=0.019)$. Compared with BVP, unipolar LBBAP resulted in further narrowing of QRSd to $123.0+-5.7$ $\mathrm{ms}(\mathrm{P}=0.006$ versus baseline and $\mathrm{P}=0.021$ versus $\mathrm{BVP})$. However, LOT-CRT resulted in more significant narrowing of QRSd to $117.0+-6.7 \mathrm{~ms}(\mathrm{P}<0.01$ versus baseline, BVP, or bipolar LBBAP).

\subsection{Follow-up}

The mean follow-up time was $296+-201$ days. Overall, LBBAP capture threshold, R-wave amplitude, and lead impedance were $0.74+-0.25 \mathrm{~V}, 13.36+-5.23 \mathrm{mV}$, and $533.73+-32.31 \Omega$ during 1-month follow-up $(\mathrm{P}>0.05$, respectively, between at the time of device implantation and at the follow-up visit). During LOT-CRT, QRSd was stable and no significant difference was observed between at the 3-month follow-up visit and at the time of device implantation $(\mathrm{P}>0.05)$. The ventricular pacing rates were $99 \%$. The latest success rate was $100 \%$. No patients showed signs of dislodgement, loss of capture, infections, embolism, or stroke associated with the implantation.

Transthoracic echocardiogram (Figure2C) evaluation data at baseline and 1-month follow-up, 3-month followup were available in all 5 patients receiving successful LOT-CRT. As shown in Table 3, the left ventricular end-diastolic dimension $(68.2 \pm 12.3 \mathrm{~mm}$ vs. $62.2 \pm 11.3 \mathrm{~mm}, \mathrm{P}=0.017)$ and LVEF $(32.8 \pm 5.2 \%$ vs. 45.0 $\pm 5.1 \%, \mathrm{P}=0.008)$ were improved at the 3 -month follow-up visit. The symptoms and the median NYHA classification score were improved significantly from $3.2 \pm 0.45$ to $2.4 \pm 0.55(\mathrm{P}=0.016)$.

\section{DISCUSSION}

\subsection{Major findings}

The present study demonstrated the following merits. (1) LOT-CRT was feasible in a small nonrandomized, nonconsecutive series of patients with reduced LVEF and LBBB. At the time of the device implantation, ECG changes during LOT-CRT were characterized with LBBB correction, narrower QRSd, and short LVAT. (2) Significant improvements in clinical and echocardiographic assessments were achieved during the mean followup period of 3 months. (3)There were no major implantation-related adverse events during perioperative period and follow-up. 


\subsection{Anatomical definition}

CRT using BVP (BVP-CRT) is as an integral part of the therapy for patients with HF with reduced LVEF and BBB, particularly LBBB ${ }^{16}$. However, up to a third of patients treated with BVP-CRT are still considered non-responders ${ }^{17}$. The reasons for BVP-CRT nonresponse are many but include LV scar burden and distribution, suboptimal LV stimulation site, sex, and limited electrical or mechanical dyssynchrony ${ }^{18}$. Patients with ischemic cardiomyopathy experience a similar response rate to BVP-CRT as their nonischemic counterparts $^{19}$. However, higher overall scar burden, a larger number of severely scarred segments, and greater scar density near the LV lead tip portend an unfavorable response to BVP-CRT in ICM patients ${ }^{20}$. There is evidence that CRT is not salutary in patients with posterolateral scar ${ }^{21}$.

\subsection{Electrophysiological definition}

Permanent LBBAP is an effective form of physiologic pacing with high success rates in patients with intact His-Purkinje conduction ${ }^{7}$. LBBAP can be a new CRT technique to correct LBBB, provide ventricular synchrony, and improve clinical symptoms with LV reverse remodeling ${ }^{22}$.

There is evident in LV activation time studies where $\mathrm{LV}$ activation time is only minimally increased in RBBB but significantly increased in $\mathrm{LBBB}^{23}$. During unipolar LBBAP, as the right ventricle is predominantly activated via myocardial conduction, RV dyssynchrony may be present compared to HBP. While it does not cause LV dyssynchrony since LV activation occurs via the His-Purkinje system. So, in patients undergoing permanent LBBAP, synchronization of delayed RV activation and normal LV activation is feasible.

\subsection{LOT-CRT advantage}

However, in patients with intraventricular block or higher overall scar burden, success rates are somewhat limited depending on the site of block and the scar burden and distribution of interventricular septum ${ }^{24}$. Intra or inter-ventricular dyssynchrony cannot be reduced utilizing LBBAP. LBBAP achieved only partial narrowing of QRSd in those patients with the baseline surface ECG of atypical LBBB morphology ${ }^{24}$. LOTCRT offers the advantage to use the LV lead in addition to LBBAP in a potential scenario of progression of conduction disease.

In patients with LBBB and cardiomyopathy, LOT-CRT resulted in significant electrical resynchronization in these patients. In our study, which included $60 \%$ of patients with severe ischemic cardiomyopathy, LOT-CRT resulted in high clinical and echocardiographic response rates. Our results indicated that those patients with LBBB and higher overall scar burden might be the desired candidates of LOT-CRT.

\subsection{Limitations}

First, it is time consuming. The duration of operation time was $152 \pm 31 \mathrm{~min}$, and the time of X-ray fluoroscopy was $26.2 \pm 5.9 \mathrm{~min}$, both are longer than previous report $(117 \pm 48 \text { and } 16.4 \pm 12.3 \mathrm{~min})^{7}$. Second, this study includes a small sample in a single center. Third, this study had a short follow-up interval. We expect long-term favorable clinical benefits. Furthermore, this study enrolled only 3 ischemic patients. Although this uncontrolled nonrandomized study does not provide sufficient data to support this conclusion, we observed significant echocardiographic and clinical improvement in these patients with HF treated with LOT-CRT.

\section{CONCLUSIONS}

The study demonstrates that LOT-CRT is clinically feasible in patients with systolic HF and LBBB. LOTCRT was associated with significant narrowing of QRS duration and improvement in LV function, especially in patients with ICM.

\section{Conflict of interest statement}

The authors declare no Conflict of Interest for this article.

\section{Funding}


None.

\section{Acknowledgments}

Not applicable.

\section{Data Availability Statement}

Datasets analyzed in this study are available from the corresponding author upon reasonable request due to privacy or other restrictions.

\section{References}

1. Dotsenko O, Barsheshet A, Huang DT. Cardiac resynchronization therapy for prevention of heart failure events in elderly patients with left ventricular dysfunction. Expert Rev Cardiovasc Ther. 2012;10(10):13191327.

2. Vijayaraman P, Panikkath R, Mascarenhas V, Bauch TD. Left Bundle Branch Pacing Utilizing Three Dimensional Mapping. J Cardiovasc Electrophysiol. 2019.

3. Sharma PS, Dandamudi G, Herweg B, et al. Permanent His-bundle pacing as an alternative to biventricular pacing for cardiac resynchronization therapy: A multicenter experience.Heart Rhythm. 2018;15(3):413-420.

4. Sharma PS, Naperkowski A, Bauch TD, et al. Permanent His Bundle Pacing for Cardiac Resynchronization Therapy in Patients With Heart Failure and Right Bundle Branch Block. Circ Arrhythm Electrophysiol. 2018;11(9):e006613.

5. Li X, Li H, Ma W, et al. Permanent left bundle branch area pacing for atrioventricular block: Feasibility, safety, and acute effect. Heart Rhythm. 2019.

6. Li X, Li H, Ma W, et al. Permanent left bundle branch area pacing for atrioventricular block: Feasibility, safety, and acute effect. Heart Rhythm. 2019;16(12):1766-1773.

7. Vijayaraman P, Subzposh FA, Naperkowski A, et al. Prospective evaluation of feasibility and electrophysiologic and echocardiographic characteristics of left bundle branch area pacing. Heart Rhythm. 2019;16(12):1774-1782.

8. Strauss DG, Selvester RH, Wagner GS. Defining left bundle branch block in the era of cardiac resynchronization therapy. Am J Cardiol. 2011;107(6):927-934.

9. Mafi-Rad M, Luermans JG, Blaauw Y, et al. Feasibility and Acute Hemodynamic Effect of Left Ventricular Septal Pacing by Transvenous Approach Through the Interventricular Septum. Circ Arrhythm Electrophysiol. 2016;9(3):e003344.

10. Chen K, Li Y, Dai Y, et al. Comparison of electrocardiogram characteristics and pacing parameters between left bundle branch pacing and right ventricular pacing in patients receiving pacemaker therapy. Europace.2019;21(4):673-680.

11. Li X, Li H, Ma W, et al. Permanent left bundle branch area pacing for atrioventricular block: feasibility, safety and acute effect. Heart rhythm. 2019.

12. Huang W, Chen X, Su L, Wu S, Xia X, Vijayaraman P. A beginner's guide to permanent left bundle branch pacing. Heart Rhythm. 2019.

13. Feng XF, Zhang PP, Liu B, Zhao Y, Lu QF, Li YG. Permanent left bundle branch area pacing utilizing intracardiac echocardiogram. BMC Cardiovasc Disord.2020;20(1):377.

14. Vijayaraman P, Panikkath R, Mascarenhas V, Bauch TD. Left bundle branch pacing utilizing three dimensional mapping. J Cardiovasc Electrophysiol.2019;30(12):3050-3056. 
15. Vijayaraman P, Dandamudi G, Zanon F, et al. Permanent His bundle pacing: Recommendations from a Multicenter His Bundle Pacing Collaborative Working Group for standardization of definitions, implant measurements, and follow-up.Heart Rhythm. 2018;15(3):460-468.

16. Cleland JG, Daubert JC, Erdmann E, et al. The effect of cardiac resynchronization on morbidity and mortality in heart failure. N Engl J Med. 2005;352(15):1539-1549.

17. Singh JP, Klein HU, Huang DT, et al. Left ventricular lead position and clinical outcome in the multicenter automatic defibrillator implantation trial-cardiac resynchronization therapy (MADIT-CRT) trial. Circulation.2011;123(11):1159-1166.

18. Vijayaraman P, Herweg B, Ellenbogen KA, Gajek J. His-Optimized Cardiac Resynchronization Therapy to Maximize Electrical Resynchronization: A Feasibility Study.Circ Arrhythm Electrophysiol. 2019;12(2):e006934.

19. Molhoek SG, Bax JJ, van Erven L, et al. Comparison of benefits from cardiac resynchronization therapy in patients with ischemic cardiomyopathy versus idiopathic dilated cardiomyopathy. Am J Cardiol. 2004;93(7):860-863.

20. Adelstein EC, Saba S. Scar burden by myocardial perfusion imaging predicts echocardiographic response to cardiac resynchronization therapy in ischemic cardiomyopathy. Am Heart J. 2007;153(1):105-112.

21. Bleeker GB, Kaandorp TA, Lamb HJ, et al. Effect of posterolateral scar tissue on clinical and echocardiographic improvement after cardiac resynchronization therapy. Circulation. 2006;113(7):969-976.

22. Zhang W, Huang J, Qi Y, et al. Cardiac resynchronization therapy by left bundle branch area pacing in patients with heart failure and left bundle branch block. Heart Rhythm. 2019;16(12):1783-1790.

23. Varma N. Left ventricular conduction delays and relation to QRS configuration in patients with left ventricular dysfunction. Am J Cardiol.2009;103(11):1578-1585.

24. Li X, Qiu C, Xie R, et al. Left bundle branch area pacing delivery of cardiac resynchronization therapy and comparison with biventricular pacing. ESC Heart Fail.2020;7(4):1711-1722.

\section{Table and Figure Legends}

Table 1 . Baseline characteristics of patients for LBBAP with a CRT device

LBBAP, left bundle branch area pacing; ICM, ischemic cardiomyopathy; AF, atrial fibrillation; LVEDD, left ventricular end-diastolic dimension; LVEF, left ventricular ejection fraction

Table 2. Pacing parameters of LOT-CRT during the procedure

$L B B A P$, left bundle branch area pacing; $L V$, left ventricle; $R V$, right ventricle

Table 3. Comparison of the pre- and post-operation TTE parameters (mean \pm SD)

LVEDD, left ventricular end diastolic diameter, LVEF, left ventricular ejection fraction

Figure 1. Left-bundle-branch-optimized cardiac resynchronization therapy in a patient with ischemic cardiomyopathy.

A, Baseline ECG shows LBBB with QRS duration of $160 \mathrm{~ms}$. B, During pacing with LOT-CRT, left bundle branch block correction pattern with QRS duration of $115 \mathrm{~ms}$ is seen.

Figure 2. Fluoroscopic image and echo image of LOT-CRT in a patient with ischemic cardiomyopathy

$\mathrm{A}$ and $\mathrm{B}$ Fluoroscopic image in the LAO $40^{\circ}$ and RAO $30^{\circ}$ projection. These images showed the final lead position in the IVS. RA, right atrial lead; LV, left ventricular lead; LBB, left bundle branch lead; RD, right defibrillator lead. 
C Intracardiac echocardiography image. Parasternal short-axis view demonstrating the depth of the lead in the interventricular septum. Arrow, left bundle branch lead.
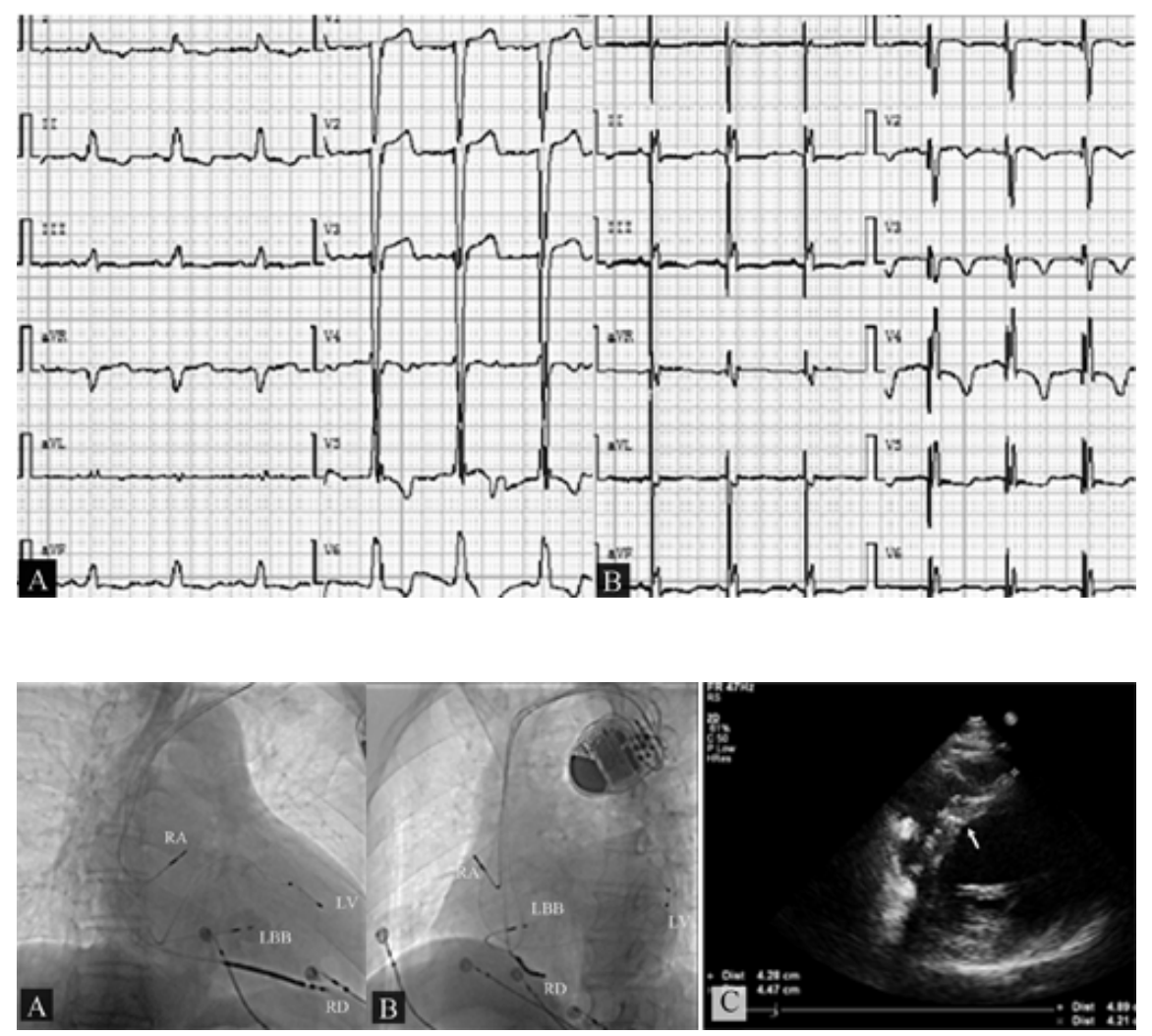

\section{Hosted file}

Table 1.pdf available at https://authorea.com/users/380844/articles/496657-cardiacresynchronization-therapy-using-left-bundle-branch-area-and-left-ventricular-pacing

Hosted file

table 2.rtf available at https://authorea.com/users/380844/articles/496657-cardiacresynchronization-therapy-using-left-bundle-branch-area-and-left-ventricular-pacing

\section{Hosted file}

table 3.rtf available at https://authorea.com/users/380844/articles/496657-cardiacresynchronization-therapy-using-left-bundle-branch-area-and-left-ventricular-pacing 